\title{
Enhancement of pinch force in the lower leg by anodal transcranial direct current stimulation
}

\author{
Satoshi Tanaka • Takashi Hanakawa - Manabu Honda • \\ Katsumi Watanabe
}

Received: 16 March 2009 / Accepted: 13 May 2009 / Published online: 29 May 2009

(C) The Author(s) 2009. This article is published with open access at Springerlink.com

\begin{abstract}
Transcranial direct current stimulation (tDCS) is a procedure to polarize human brain. It has been reported that $\mathrm{tDCS}$ over the hand motor cortex transiently improves the performance of hand motor tasks. Here, we investigated whether tDCS could also improve leg motor functions. Ten healthy subjects performed pinch force (PF) and reaction time (RT) tasks using the left leg before, during and after anodal, cathodal or sham tDCS over the leg motor cortex.
\end{abstract}

S. Tanaka $\cdot$ K. Watanabe

ERATO Shimojo Implicit Brain Function Project,

Japan Science and Technology Agency (JST),

Atsugi, Kanagawa 243-0198, Japan

S. Tanaka $(\bowtie) \cdot \mathrm{K}$. Watanabe

Cognitive Science Laboratory,

Research Center for Advanced Science and Technology,

University of Tokyo, 4-6-1, Komaba, Meguro-ku,

Tokyo 153-8904, Japan

e-mail: tanaka@fennel.rcast.u-tokyo.ac.jp

S. Tanaka $\cdot$ T. Hanakawa $\cdot$ M. Honda

Department of Cortical Function Disorders,

National Institute of Neuroscience,

Kodaira Tokyo 187-8502, Japan

T. Hanakawa

PRESTO, JST, Kawaguchi, Saitama 332-0012, Japan

M. Honda

CRESTO, JST, Kawaguchi, Saitama 332-0012, Japan

K. Watanabe

Institute for Human Science and Biomedical Engineering,

National Institutes of Advanced Science and Technology,

Tsukuba, Ibaraki 305-8566, Japan
The anodal tDCS transiently enhanced the maximal leg PF but not RT during its application. Neither cathodal nor sham stimulation changed the performance. None of the interventions affected hand PF or RT, showing the spatial specificity of the effect of tDCS. These results indicate that motor performance of not only the hands but also the legs can be enhanced by anodal tDCS. tDCS may be applicable to the neuro-rehabilitation of patients with leg motor disability.

Keywords Gait $\cdot$ Leg $\cdot$ Motor cortex $\cdot$ Rehabilitation · Transcranial direct current stimulation - Transcranial magnetic stimulation

\section{Introduction}

Transcranial direct current stimulation (tDCS) is a recently proposed technique that stimulates brain regions by delivering weak direct currents through the skull. Depending on the polarity of stimulation, it can increase or decrease the excitability of a stimulated cortical region. The excitability of the hand motor cortex, for example, is transiently increased by anodal tDCS and decreased by cathodal tDCS (Nitsche and Paulus 2000; Nitsche and Paulus 2001; Furubayashi et al., 2008). Furthermore, tDCS-induced excitability changes are associated with changes in the performance of both cognitive and motor tasks (Priori 2003; Wassermann and Grafman 2005; Tanaka and Watanabe 2009). Because a tDCS device is relatively small and elicits no acoustic noise and muscle twitching compared with other brain stimulation techniques, it is suitable for double-blind sham-controlled studies and clinical applications (Gandiga et al. 2006; Hummel and Cohen 2006; Fregni and PascualLeone 2007). 
Previous studies have shown that tDCS can enhance the motor performance of the hands. For example, in both healthy volunteers and stroke patients the performance of hand motor tasks mimicking activities of daily living (Jebsen-Taylor hand function test) temporarily improved when anodal tDCS was applied to the hand motor cortex at an intensity of $1 \mathrm{~mA}$ for $20 \mathrm{~min}$ (Fregni et al. 2005; Hummel et al. 2005; Hummel and Cohen 2005; Boggio et al. 2006). Anodal tDCS over the hand motor cortex also increased maximal pinch force (PF) and shortened reaction time (RT) in stroke patients performing simple hand motor tasks (Hummel and Cohen 2005; Hummel et al. 2006). These findings imply that tDCS could be powerful tool for modulating hand function and might contribute to motor recovery in neuro-rehabilitative settings.

Many neurological disorders and strokes often lead to marked deficits in leg motor function and gait, and patients suffer from leg disabilities and gait disturbances such as impaired force generation, lower gait velocity, decreased cadence and shorter step length. Although effects of tDCS on leg motor functions would thus be relevant to neurorehabilitation after neurological disorders and stroke, whether tDCS applied over the leg motor cortex can have any effect on leg motor performance is still unknown. We therefore examined whether application of tDCS centered over the leg motor cortex changed PF and RT in healthy subjects. A previous study demonstrated that anodal tDCS transiently enhanced the motor-evoked potentials (MEPs) in the contralateral leg motor cortex in healthy subjects (Jeffery et al. 2007), and we hypothesized that anodal tDCS over the leg motor cortex could facilitate the performance of leg motor tasks.

\section{Methods}

\section{Subjects}

Ten healthy adult volunteers ( 8 male and 2 female; mean age 23.8 years; range $20-35$ years) participated in the present study. All reported right hand dominant and right leg dominant, and none had a history of psychiatric or neurological illness. All subjects gave written, informed consent before the experiments, which were approved by the local ethics committee of the National Institute for Neuroscience.

\section{Procedure}

We examined the effect of tDCS over the right leg motor cortex on the PF and RT tasks (Fig. 1a). In the PF task, maximal pinch forces of the left toe and the left finger were measured. In the RT task, subjects brought the left toe up (ankle flexion) or extended the left thumb (finger extension)

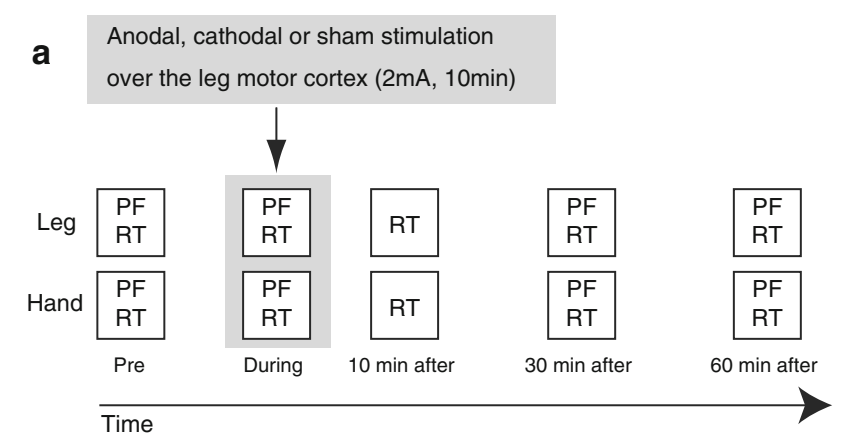

b

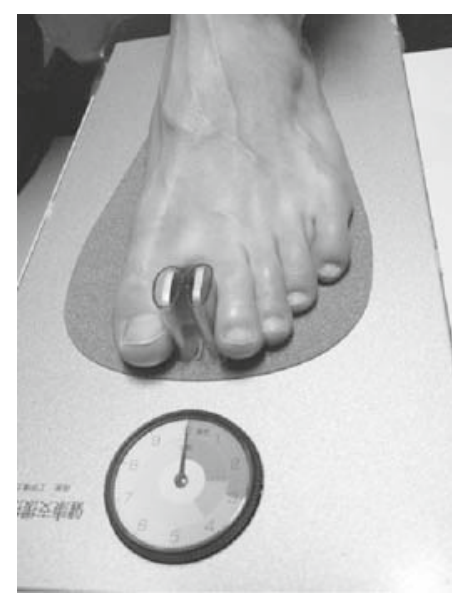

Fig. 1 a Experimental design. The effect of tDCS on the leg motor cortex was examined in the PF and RT tasks. In the PF task the maximal pinch strength of the left leg was measured. In the RT task the subjects responded to a visual stimulus by using the left leg as quickly as possible. All subjects participated in anodal-tDCS, cathodal-tDCS and sham-stimulation experimental sessions, and each session consisted of four blocks of the PF task and five blocks of the RT task. After subjects performed a block of the PF and RT tasks, 2 mA tDCS over the leg motor cortex was applied for $10 \mathrm{~min}$. The spatial focality of tDCS effect was evaluated by also measuring the PF and RT when the tasks were performed with the left hand. b Leg pinch force device. Maximal PF of the left great toe and the digitus secundus was assessed using a toe-gap force measurement device. Subjects were seated in an armchair and asked to squeeze the gauge as hard as possible for $1-3 \mathrm{~s}$

as quickly as possible in response to a visual stimulus presented on a computer screen. All subjects participated in three sessions: anodal, cathodal tDCS and sham stimulation. The three sessions were separated by at least 1 week and the order was counter-balanced among subjects. Before starting each session, the subjects practiced the tasks for familiarization. Each session consisted of four blocks of the PF task (before, during, 30 and 60 min after the tDCS intervention: 3 trials per block) and the five blocks of RT task (before, during, 10, 30 and $60 \mathrm{~min}$ after the offset of the intervention: 25 trials per block). It took roughly $1.5 \mathrm{~min}$ to complete a block of the PF task, and 3 min to complete a block of the RT task. In all tasks, the non-dominant (i.e., left) hand and leg were used to avoid ceiling effect. The orders of the leg and hand use and of the PF and RT tasks 
were counter-balanced across the subjects. No feedback regarding PF and RT performances was given. One subject performed only the leg PF task and another performed only the leg PF and RT tasks. The hand RT task data for another subject were not collected because of machine trouble.

Tasks

\section{Leg pinch force measurement}

Maximal PF (in newtons) of the left great toe and the digitus secundus was assessed using a toe-gap force measurement device (Fig. 1b; Sokushi Checker, Shinkikaku Shuppan, Tokyo, Japan). Muscles directly related to PF are the transverse head of adductor hallucis and the plantar interossei (Yamashita et al. 2006). Toe PF is also generated by collaborative activation of other muscles, such as the extensor digitorum brevis, extensor digitorum longus, flexor digitorum brevis and tibialis anterior (TA). The electromyogram activities of these muscles during the PF measurements increased with the generated force (Yamashita and Saito 2002). The measured PF is closely related to the physical performance of the lower limbs (e.g., 10-m walking time; Yamashita et al. 2006). The PF is therefore considered to reflect the muscular power of the lower limb.

During the measurement the subjects were seated in an armchair with the left leg flexed at the hip $\left(90^{\circ}\right)$ and the knee $\left(90^{\circ}\right)$ and with the ankle in $90^{\circ}$ of plantar flexion. The subjects used their left great toe and digitus secundus to squeeze the gauge as hard as possible for $1-3 \mathrm{~s}$. The subjects were instructed not to raise their heel during the measurement. The number of trials ( 3 trials) per block was determined to avoid the effect of fatigue. No significant differences of baseline PF performance among three (anode, cathode and sham) conditions (see "Results") suggest the test-retest reliability on this way of the measurement. All measurements were performed by the same investigator, with the same device, and under the same experimental conditions.

\section{Leg reaction time task}

The subjects were seated in front of a computer monitor. Each trial started with a visual warning signal (green square) on the center of the screen, after which a GO signal (red square) was presented at random intervals (2$6 \mathrm{~s})$. Subjects were asked to relax the muscle immediately after they responded and to get ready for the next trial. Subjects used the left leg to keep pressing a pedal (FS30A-USB-UL, Kinesis Corporation, Bothell, WA, USA) while the warning signal was presented, and they responded to the GO signal by releasing the pedal as quickly as possible. The RT was defined as the interval (ms) between the onset of the GO signal and the onset of the release movement.

\section{Hand pinch force measurement}

The subjects were seated in the chair with both arms relaxed. They held the arm of a dynamometer (Hydraulic Hand Dynamometer, Baseline, Irvington, NY, USA) between the lateral aspect of the middle phalanx of the index finger and the thumb pad of the left hand and squeezed it as hard as possible for 1-3 s (Mathiowetz et al. 1984, 1985; Muellbacher et al. 2001; Hummel et al. 2006).

\section{Hand reaction time task}

The overall procedure was the same as that of the leg RT task, but with a hand-made button box instead of the foot pedal. The subjects used their left thumb to keep pressing the button while the warning signal was presented, and they responded to the GO signal by releasing the button as quickly as possible.

\section{tDCS}

A DC Stimulator Plus (NeuroConn, Ilmenau, Germany) delivered direct current through two sponge surface electrodes (each with a surface area of $35 \mathrm{~cm}^{2}$ ) soaked with $15 \mathrm{mM} \mathrm{NaCl}$ (Dundas et al. 2007). One electrode was positioned over the left leg representation of the right motor cortex in which transcranial magnetic stimulation (TMS) elicited twitches in the TA, and the other electrode was placed on the forehead above the contralateral orbit. The intensity of anodal and cathodal tDCS was $2 \mathrm{~mA}$. The tDCS was applied for 10 min (including the initial $15 \mathrm{~s}$ during which the current was gradually increased from 0 and the last $15 \mathrm{~s}$ during which it was gradually decreased to 0 ). Anodal tDCS with these parameters has been reported to change the excitability of the leg motor cortex without inducing side effects (Jeffery et al. 2007). The current density at the stimulation electrodes was $0.057 \mathrm{~mA} / \mathrm{cm}^{2}$ and the total charge was $0.069 \mathrm{C} / \mathrm{cm}^{2}$. These parameters are in accordance with a safety criterion and far below the threshold for tissue damage (Nitsche et al. 2003). The voltage during tDCS application was less than $10 \mathrm{~V}$. For sham stimulation the same procedure was used but current was applied for only $30 \mathrm{~s}$ (Gandiga et al. 2006).

\section{Transcranial magnetic stimulation}

The position of tDCS electrode was centered on the scalp position where TMS elicited twitching of the left TA. TMS was delivered using A Magstim 200 Stimulator (Magstim, Whitland, UK) through $80-\mathrm{mm}$ figure-eight coils oriented 
in the posterior-anterior direction. At the beginning of each session, we determined the resting motor threshold (rMT) for the left TA muscle over the leg motor cortex in the right hemisphere. rMT was defined as the lowest intensity of TMS output required to elicit MEPs of $100-\mu \mathrm{V}$ peak-topeak amplitude in five of ten consecutive trials (Rossini et al. 1994). We determined the optimal position for activation of the TA muscle by moving the coils in $1 \mathrm{~cm}$ steps along the mid-sagittal line crossing $\mathrm{Cz}$ (International 10-20 system). Using this method, TMS was able to elicit leg MEPs in all subjects. The mean rMT was $80.9 \pm 9.2$ (mean $\pm \mathrm{SD}$ ) percent of maximum stimulator output. EMG surface electrodes were positioned on the skin overlying the left TA muscles in a bipolar montage (inter-electrode distance $2 \mathrm{~cm}$ ).

\section{Data analysis}

The data of the four tasks (leg PF, leg RT, hand PF and hand RT) were analyzed separately. For each subject, the maximal PF out of three measurements and the median RT of 25 trials were calculated for each block. The data were normalized with respect to the performances before the intervention. For each task, the normalized data were subjected to a two-way analysis of variance (ANOVA) with intervention (anodal, cathodal and sham) and TIME (time course during and after current stimulation: three time points for the PF tasks and four for the RT tasks) as withinsubject factors. Post hoc tests were performed with Bonferroni correction for multiple comparisons.

\section{Results}

No subjects reported any side effects. None noticed the difference between real and sham stimulation sessions. One-way ANOVA revealed that there was no significant difference in pre-stimulation blocks in all four tasks [leg PF $\left(F_{(2,18)}=0.04\right.$, n.s. $)$, leg RT $\left(F_{(2,16)}=0.01\right.$, n.s. $)$, hand PF $\left(F_{(2,14)}=0.26\right.$, n.s. $)$ and hand $\mathrm{RT}\left(F_{(2,12)}=1.18\right.$, n.s. $\left.)\right]$. The data were therefore normalized with respect to the performances before the intervention for each subject and each task (Fig. 2a).

\section{Leg motor tasks}

The effects of tDCS on the maximal PF in the leg motor tasks were evaluated by performing a two-way repeated measure of ANOVA with factors of intervention (anodal, cathodal and sham) and TIME (during, 30 and 60 min after stimulation) (Fig. 2a, left upper). The main effects of TIME $\left[F_{(2,18)}=0.44\right.$, n.s. $]$ and the intervention $\times$ time interaction $\left[F_{(4,36)}=2.36, P=0.71\right]$ were not significant, whereas the main effect of the intervention was significant $\left[F_{(2,18)}=6.87\right.$, $P<0.01]$. The significant main effect of the intervention indicated that the three types of tDCS influenced maximal leg PF differently. Post hoc analyses revealed that the maximal PF during anodal tDCS was significantly higher compared to sham $(P<0.05$ with Bonferroni correction) and cathodal tDCS $(P<0.001)$. Thirty minutes after the offset of current flow, the maximal PF was still significantly higher for anodal than for cathodal tDCS $(P<0.01)$ while there was no longer significant difference between anodal and sham stimulation. There were no significant differences in the maximal PF $60 \mathrm{~min}$ after stimulation. Individual maximal leg PF values before and during tDCS application are shown in Fig. 2b, where one sees that with anodal tDCS the maximal PF increased in all subjects (pre vs. during comparison, paired $t$ test, $\left.t_{(9)}=5.61, P<0.001\right)$ and with cathodal and sham stimulation there was no consistent change across subjects [cathodal $\left(t_{(9)}=0.75\right.$, n.s.), sham $\left(t_{(9)}=0.25\right.$, n.s. $\left.)\right]$. These results indicated that anodal tDCS transiently enhanced maximal leg PF during and $30 \mathrm{~min}$ after its application but that neither cathodal tDCS nor sham stimulation affected maximal leg PF significantly. These findings are consistent with a previous report that anodal tDCS enhanced the excitability of the leg motor cortex (Jeffery et al. 2007). On the other hand, none of the interventions had a significant effect on leg RT [Fig. 2a left lower, intervention $\left(F_{(2,16)}=0.20\right.$, n.s. $)$ and time $\left(F_{(3,24)}=0.63\right.$, n.s. $)$, interaction $\left(F_{(6,48)}=0.56\right.$, n.s. $\left.)\right]$.

Hand motor tasks

None of the interventions significantly affected the maximal $\mathrm{PF}$ in the hand motor tasks (Fig. 2a right upper, intervention $\left(F_{(2,14)}=0.34\right.$, n.s. $)$, time $\left(F_{(2,14)}=1.62\right.$, n.s. $)$, interaction $\left(F_{(4,28)}=0.39\right.$, n.s. $)$ and RT [Fig. 2a right lower, intervention $\left(F_{(2,12)}=0.60\right.$, n.s. $)$, time $\left(F_{(3,18)}=0.32\right.$, n.s. $)$, interaction $\left(F_{(6,36)}=0.86\right.$, n.s. $\left.)\right]$. The absence of significant effects of tDCS on either the PF or the RT of the hand indicates that the anodal tDCS with the present stimulation parameters had somatotopically specific effects.

\section{Discussion}

The present results demonstrated for the first time that anodal tDCS over the leg motor cortex temporarily facilitated maximal leg PF contralateral to the stimulation. The enhancement lasted for $30 \mathrm{~min}$ after the end of stimulation and was not observed in the hand motor tasks. The effecter-specificity of the modulation indicated that the results were not caused by general effects such as increased arousal or changes in attention, motivation or mood. The effecter-specificity also indicates that tDCS 
Fig. 2 a Results showing the effect of tDCS on PF and RT tasks. Mean performance is plotted as a function of time relative to the intervention, with bars indicating SEM $(* P<0.05$, $* * P<0.01)$. Leg PF (left upper), leg RT (left lower), hand PF (right upper), Hand RT (right lower). The data are normalized with respect to baseline value before the intervention. Anodal tDCS (grey circle) significantly increased the maximal PF during its application and $30 \mathrm{~min}$ after stimulation compared to baseline. Neither cathodal (white rectangle) nor sham (black triangle) stimulation changed the maximal PF, and neither anodal nor cathodal significantly affect on leg RT, hand PF or hand RT. b Individual results for maximal leg PF. Each of the plotted values plot is an individual maximal PF before or during tDCS application. Anodal tDCS increased the PF maximal in all subjects, and neither cathodal tDCS nor sham stimulation affected $\mathrm{PF}$ consistently across subjects $(* * * P<0.001)$
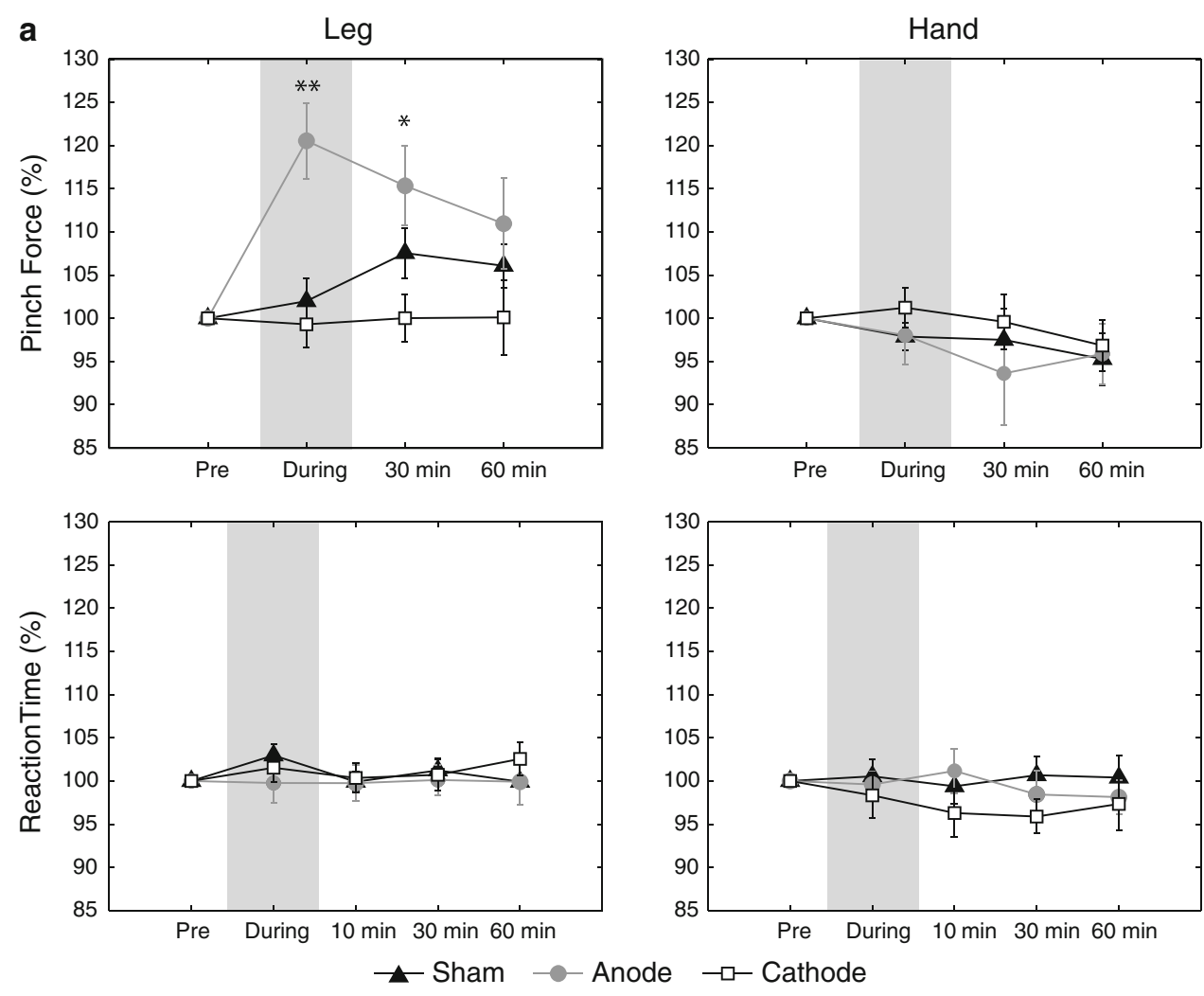

b
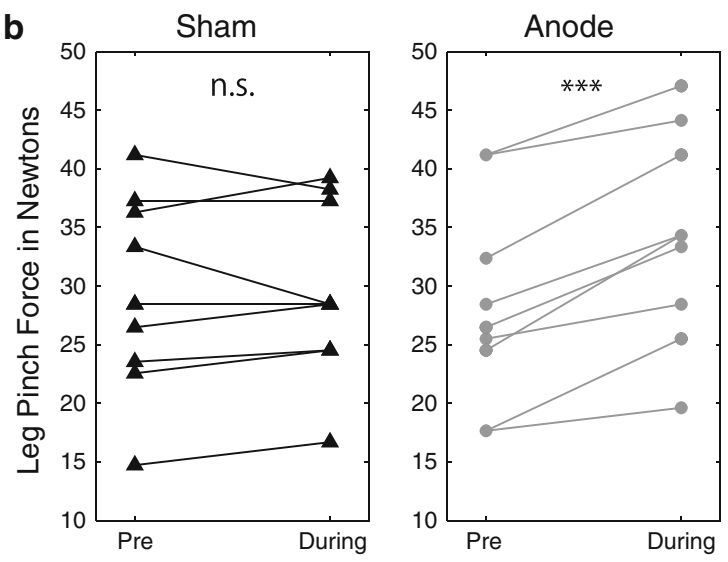

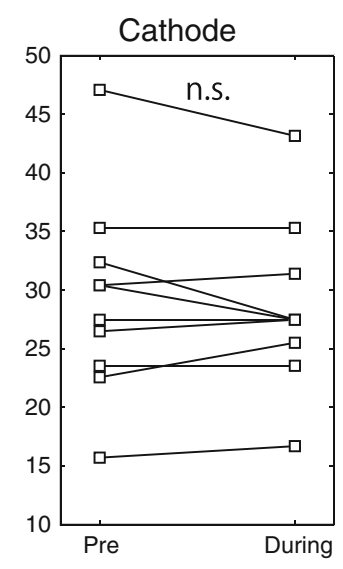

could induce the change in the leg motor function without affecting the hand function, presumably because the hand motor cortex is about $3-4 \mathrm{~cm}$ apart from the leg motor cortex.

Hand motor performance enhanced by anodal tDCS has been observed in previous tDCS studies (Hummel et al. 2005, 2006), and the degree of the improvement of leg PF in our study $(20.5 \%)$ was comparable to the degree of tDCS-induced improvement observed in previous studies of hand motor function. In those studies, the current was $1 \mathrm{~mA}$, whereas it was of $2 \mathrm{~mA}$ in our study because the leg motor areas are less excitable than hand motor areas (Jeffery et al. 2007). Therefore, there might be the qualitatively similar effects on behavioral performance between 1-mA anodal
tDCS over the hand motor cortex and 2-mA anodal tDCS over the leg motor cortex.

In the present study, the anodal tDCS over the leg motor cortex did not change the leg RT contralateral to the stimulation. The RT in hand motor tasks has been reported to be facilitated by anodal tDCS (Hummel and Cohen 2005; Hummel et al. 2006). The absence of an effect of tDCS on leg RT might be due to performance ceiling. In the present study, the subjects were healthy adult volunteers (mean age 23.8 years) and their RTs were already quite short before the intervention (mean RT $272 \mathrm{~ms}$ ). The subjects in the previous studies, in contrast, were older patients (mean age 57.0 years) with chronic stroke and might therefore be expected to respond more slowly than younger healthy 
adults (Hummel et al. 2006). Another possibility is that, because of low spatial focality of tDCS, anodal tDCS in the previous studies stimulated not only the hand motor cortex but also the premotor cortex. Then, anodal tDCS may facilitate externally triggered movement, a possible function of the premotor cortex (Goldberg 1985; Wessel et al. 1997; Crosson et al. 2001), which is required for performing the RT task. It is also possible that the RT task might not be sensitive enough for detecting the effect of tDCS, or the stimulation strength and/or duration (10 $\mathrm{min}$ of $2 \mathrm{~mA}$ ) might not be sufficient to induce meaningful behavioral gains in the task.

In accordance with tDCS effect on the excitability of leg motor cortex (Jeffery et al. 2007), we did not observe significant effect of cathodal tDCS on the behavioral performances. This might be due to the leg motor cortex having fewer inhibitory circuits than the hand motor cortex, or cathodal current might be less effective in the leg motor cortex because of the different orientation and position of the leg motor cortex relative to the scalp (Jeffery et al. 2007). One difference between the results of the electrophysiological study by Jeffery et al. (2007) and our present results is the duration of the after-effect of tDCS. In their study, 10 min of anodal tDCS increased the excitability of the leg cotricospinal tract for over $60 \mathrm{~min}$ after the stimulation. The effect of anodal tDCS on the maximal PF of the leg that we observed, on the other hand, was relatively short-lasting, and the PF returned to its baseline value 60 min after the stimulation. It might be more difficult to induce long-lasting change of final behavioral output by tDCS. Alternatively, this discrepancy might be due to the differences in equipment, subject population and/or experimental conditions between Jeffery et al. (2007) and ours.

Mechanisms underlying the enhanced leg PF are still speculative. One possibility is that an increase of corticospinal excitability by anodal tDCS in the leg motor cortex contributed to the behavioral gain. Another possibility is that tDCS increase intermuscular coupling. Anodal tDCS over the hand motor cortex has been reported to increase beta-band intermuscular coherence in the first dorsal interosseous and extensor digitorum communis muscles (Power et al. 2006). Collaborative activation of lower limb muscles is needed for generation of the PF in toes. Thus, a more optimal coupling of these muscles by anodal tDCS may contribute to the enhanced maximal PF in the leg. Resolving this issue will require further experiments in which leg behavioral performance and brain and muscular activities are measured simultaneously and correlated.

In summary, we have shown that the leg motor performance of healthy adult subjects is transiently enhanced by anodal tDCS over the contralateral leg motor cortex. This is the direct evidence that tDCS can induce not only the change of local cortical excitability (physiological changes) but also behavioral gain of the motor function (behavioral/ functional changes). Because tDCS can be applied while subjects are performing a leg motor task, it might be useful in the neuro-rehabilitation of patients with leg motor disabilities.

Acknowledgments This work was supported by ERATO Shimojo Implicit Brain Project to ST and KW and KAKENHI (20033030) to TH. We thank to Dr. Shinsuke Shimojo and Kei Omata for their helpful comments.

Open Access This article is distributed under the terms of the Creative Commons Attribution Noncommercial License which permits any noncommercial use, distribution, and reproduction in any medium, provided the original author(s) and source are credited.

\section{References}

Boggio PS, Castro LO, Savagim EA, Braite R, Cruz VC, Rocha RR, Rigonatti SP, Silva MT, Fregni F (2006) Enhancement of nondominant hand motor function by anodal transcranial direct current stimulation. Neurosci Lett 404:232-236

Crosson B, Sadek JR, Maron L, Gokcay D, Mohr CM, Auerbach EJ, Freeman AJ, Leonard CM, Briggs RW (2001) Relative shift in activity from medial to lateral frontal cortex during internally versus externally guided word generation. J Cogn Neurosci 13:272-283

Dundas JE, Thickbroom GW, Mastaglia FL (2007) Perception of comfort during transcranial DC stimulation: effect of $\mathrm{NaCl}$ solution concentration applied to sponge electrodes. Clin Neurophysiol 118:1166-1170

Fregni F, Pascual-Leone A (2007) Technology insight: noninvasive brain stimulation in neurology-perspectives on the therapeutic potential of rTMS and tDCS. Nat Clin Pract Neurol 3:383-393

Fregni F, Boggio PS, Mansur CG, Wagner T, Ferreira MJ, Lima MC, Rigonatti SP, Marcolin MA, Freedman SD, Nitsche MA, PascualLeone A (2005) Transcranial direct current stimulation of the unaffected hemisphere in stroke patients. NeuroReport 16:15511555

Furubayashi T, Terao Y, Arai N, Okabe S, Mochizuki H, Hanajima R, Hamada M, Yugeta A, Inomata-Terada S, Ugawa Y (2008) Short and long duration transcranial direct current stimulation (tDCS) over the human hand motor area. Exp Brain Res 185:279-286

Gandiga PC, Hummel FC, Cohen LG (2006) Transcranial DC stimulation (tDCS): a tool for double-blind sham-controlled clinical studies in brain stimulation. Clin Neurophysiol 117:845-850

Goldberg G (1985) Supplementary motor area structure and function: review and hypotheses. Behav Brain Sci 8:567-616

Hummel F, Cohen LG (2005) Improvement of motor function with noninvasive cortical stimulation in a patient with chronic stroke. Neurorehabil Neural Repair 19:14-19

Hummel FC, Cohen LG (2006) Non-invasive brain stimulation: a new strategy to improve neurorehabilitation after stroke? Lancet Neurol 5:708-712

Hummel F, Celnik P, Giraux P, Floel A, Wu WH, Gerloff C, Cohen LG (2005) Effects of non-invasive cortical stimulation on skilled motor function in chronic stroke. Brain 128:490-499

Hummel FC, Voller B, Celnik P, Floel A, Giraux P, Gerloff C, Cohen LG (2006) Effects of brain polarization on reaction times and pinch force in chronic stroke. BMC Neurosci 7:73

Jeffery DT, Norton JA, Roy FD, Gorassini MA (2007) Effects of transcranial direct current stimulation on the excitability of the leg motor cortex. Exp Brain Res 182:281-287 
Mathiowetz V, Weber K, Volland G, Kashman N (1984) Reliability and validity of grip and pinch strength evaluations. J Hand Surg 9:222-226

Mathiowetz V, Kashman N, Volland G, Weber K, Dowe M, Rogers S (1985) Grip and pinch strength: normative data for adults. Arch Phys Med Rehabil 66:69-74

Muellbacher W, Ziemann U, Boroojerdi B, Cohen L, Hallett M (2001) Role of the human motor cortex in rapid motor learning. Exp Brain Res 136:431-438

Nitsche MA, Paulus W (2000) Excitability changes induced in the human motor cortex by weak transcranial direct current stimulation. J Physiol 527:633-639

Nitsche MA, Paulus W (2001) Sustained excitability elevations induced by transcranial DC motor cortex stimulation in humans. Neurology 57:1899-1901

Nitsche MA, Liebetanz D, Lang N, Antal A, Tergau F, Paulus W (2003) Safety criteria for transcranial direct current stimulation (tDCS) in humans. Clin Neurophysiol 114:2220-2222

Power HA, Norton JA, Porter CL, Doyle Z, Hui I, Chan KM (2006) Transcranial direct current stimulation of the primary motor cortex affects cortical drive to human musculature as assessed by intermuscular coherence. J Physiol 577:795-803
Priori A (2003) Brain polarization in humans: a reappraisal of an old tool for prolonged non-invasive modulation of brain excitability. Clin Neurophysiol 114:589-595

Rossini PM, Barker AT, Berardelli A, Caramia MD, Caruso G, Cracco RQ, Dimitrijevic MR, Hallett M, Katayama Y, Lucking CH et al (1994) Non-invasive electrical and magnetic stimulation of the brain, spinal cord and roots: basic principles and procedures for routine clinical application. Report of an IFCN committee. Electroencephalogr Clin Neurophysiol 91:79-92

Tanaka S, Watanabe K (2009) Transcranial direct current stimulation-a new tool for human cognitive neuroscience. Brain Nerve 61:53-64

Wassermann EM, Grafman J (2005) Recharging cognition with DC brain polarization. Trends Cogn Sci 9:503-505

Wessel K, Zeffiro T, Toro C, Hallett M (1997) Self-paced versus metronome-paced finger movements. A positron emission tomography study. J Neuroimaging 7:145-151

Yamashita K, Saito M (2002) Evaluation of falling risk by toe-gap force on aged. Trans Soc Instrum Control Eng 38:952-957

Yamashita K, Umezawa J, Nomoto Y, Ino S, Ifukube T, Koyama H, Kawasumi M (2006) The role of toe-gap force for the evaluation of falling risk on the elderly. IFMBE Proc 14:405-408 